\title{
Endovascular stent-graft repair for thoracic aortic aneurysm associated with right-sided aortic arch
}

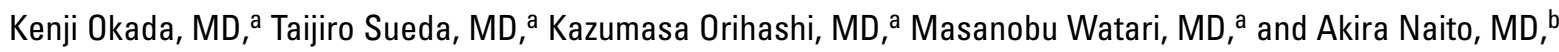 \\ Hiroshima, Japan
}

$\mathrm{R}$ ight-sided aortic arch with both left descending aorta and a retroesophageal aortic segment is an uncommon congenital vascular malformation. This abnormal anatomy is awkward to treat surgically. Recently endovascular stent-graft repair, which is less invasive than conventional surgical procedures, was applied in thoracic aortic aneurysm and showed good early results. ${ }^{1}$ We report here a case of thoracic aortic aneurysm associated with right-sided aortic arch that was successfully managed with an endovascular stent-graft.

\section{Clinical Summary}

A 76-year-old man was admitted to our hospital with a diagnosis of atherosclerotic aneurysm of the thoracic aorta associated with right-sided aortic arch. The patient had undergone coronary artery bypass grafting 10 years earlier, in which the right internal thoracic artery and a saphenous vein graft were anastomosed to the left anterior descending artery and the right coronary artery, respectively. He had been hoarse for 4 years.

Chest radiography disclosed an abnormal shadow in the upper mediastinum. Computed tomography (CT) (Figure 1, A) showed a saccular aneurysm about $6 \mathrm{~cm}$ in diameter with mural thrombus on the aortic arch and a retroesophageal aortic segment with the left descending aorta. Digital subtraction angiography (Figure 1, B) demonstrated a right-sided aortic arch and 3 cervical branches that originated from the ascending aorta in the following order: the left carotid artery, the right carotid artery, and the right subclavian artery. The left subclavian artery was occluded and the left axillary artery was opacified through a collateral root via the left vertebral artery. Echocardiography showed normal intracardiac anatomy and good left ventricular function. The diagnosis was circumflex retroesophageal aortic arch-right-sided aortic arch with left descending aorta. Transluminal endovascular grafting was indicated because the patient had undergone previous coronary bypass surgery and the left subclavian artery was already occluded.

Under general anesthesia with full hemodynamic monitoring,

\footnotetext{
From the Departments of Surgery ${ }^{\mathrm{a}}$ and Radiology, ${ }^{\mathrm{b}}$ Hiroshima University Faculty of Medicine, Hiroshima, Japan.

Received for publication Nov 6, 2000; accepted for publication Nov 13, 2000.

Address for reprints: Kenji Okada, MD, First Department of Surgery, Hiroshima University Faculty of Medicine, 1-2-3 Kasumi, Minami-ku, Hiroshima, 734-8551, Japan (E-mail: okaken@ mcai.med.hiroshima-u.ac.jp).

J Thorac Cardiovasc Surg 2001;122:185-6

Copyright $\odot 2001$ by The American Association for Thoracic Surgery

$0022-5223 / 2001 \$ 35.00+0 \quad \mathbf{1 2 / 5 4 / 1 1 3 0 1 9}$

doi: $10.1067 / \mathrm{mtc} .2001 .113019$
}

this patient was placed in the supine position. The right femoral artery was exposed and inserted with a delivery system. A handmade stent-graft composed of a modified Gianturco stainless steel Z-stent and thin-walled Dacron material was introduced into a delivery sheath catheter with a $20 \mathrm{~F}$ diameter. An angiographic catheter was put into the thoracic aorta via a guidewire from the right brachial artery. The sheath catheter was inserted through the right femoral artery and deployed with the guidance of transesophageal echocardiography and fluoroscopy. Before deployment of the stent-graft, the mean arterial pressure was lowered to $70 \mathrm{~mm}$ $\mathrm{Hg}$ with the aid of an occlusion balloon in the superior and the inferior caval veins to avoid graft migration. A second aortogram revealed a small endoleak immediately after the deployment. The postoperative course was uneventful. A small leakage from the stent-graft shown on CT 2 weeks after the intervention disappeared in the next 6 weeks (Figure 2).

\section{Comment}

Right-sided aortic arch is an uncommon congenital anomaly with an incidence of $0.1 \% .^{2}$ Thoracic aortic aneurysm associated with right-sided aortic arch is also a rare disorder, only 4 cases of surgical treatment of this disorder having been reported. ${ }^{3}$ Three anatomic findings of right-sided aortic arch make surgical treatment difficult: (1) the pattern of the aortic arch vessels, (2) the location of the descending aorta, and (3) the relation between the esophagus and the aortic arch. Caus and associates ${ }^{4}$ performed bilateral thoracotomy because the esophagus was located in front of the aortic arch and the ascending and descending aortas were in different pleural spaces. In our patient, the right internal thoracic artery, which was anastomosed to the left anterior descending artery, was positioned over the heart. Thus, either secondary median sternotomy or bilateral thoracotomy seemed to be complicated and difficult.

Endovascular stent-graft repair has become a viable therapeutic alternative in the treatment of patients with thoracic aortic aneurysm.This technique is less invasive than standard techniques and brings acceptable morbidity and mortality rates. ${ }^{1}$ The presence of a curve and branch vessels in the aortic arch makes it difficult to apply this method to aortic arch diseases. There are considered to be 3 major prerequisites for such repairs: sufficient size of the arteries providing access, limited tortuosity of the distal aortic arch, and proximal neck morphology. Specifically, suitability entails relatively straight portions of the aorta and the absence of important side branches.

In the present patient, the distal part of the aortic arch was relatively straight, the 3 cervical branches had arisen from the ascending aorta, and the left subclavian artery was totally occluded. These anatomic features made the landing zone between the right subclavian artery and the aneurysm long enough to develop a stent-graft. 

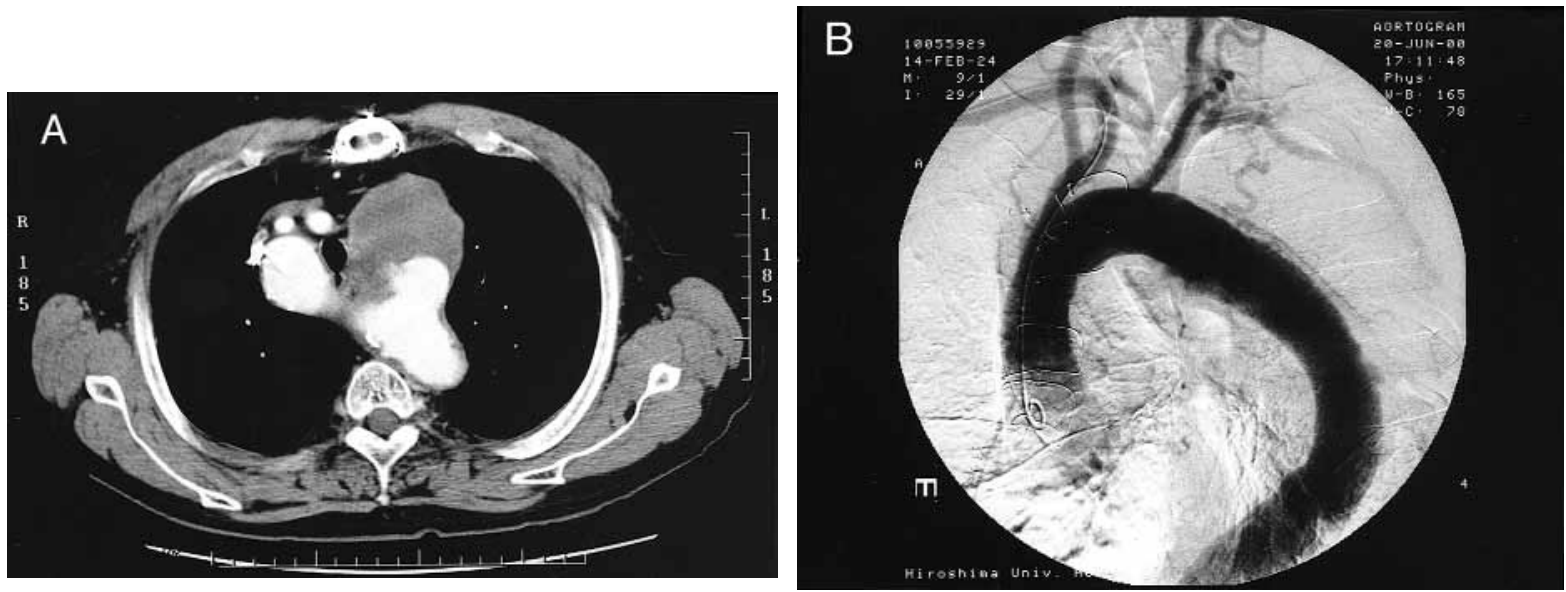

Figure 1. Preoperative CT (A) showed a saccular aneurysm about $6 \mathrm{~cm}$ in diameter on the aortic arch and a retroesophageal aortic segment with the left descending aorta. Digital subtraction angiography (B) demonstrated 3 cervical branches originating from the ascending aorta and occlusion of the left subclavian artery. The left axillary artery was opacified through collateral blood flow from the left vertebral artery.
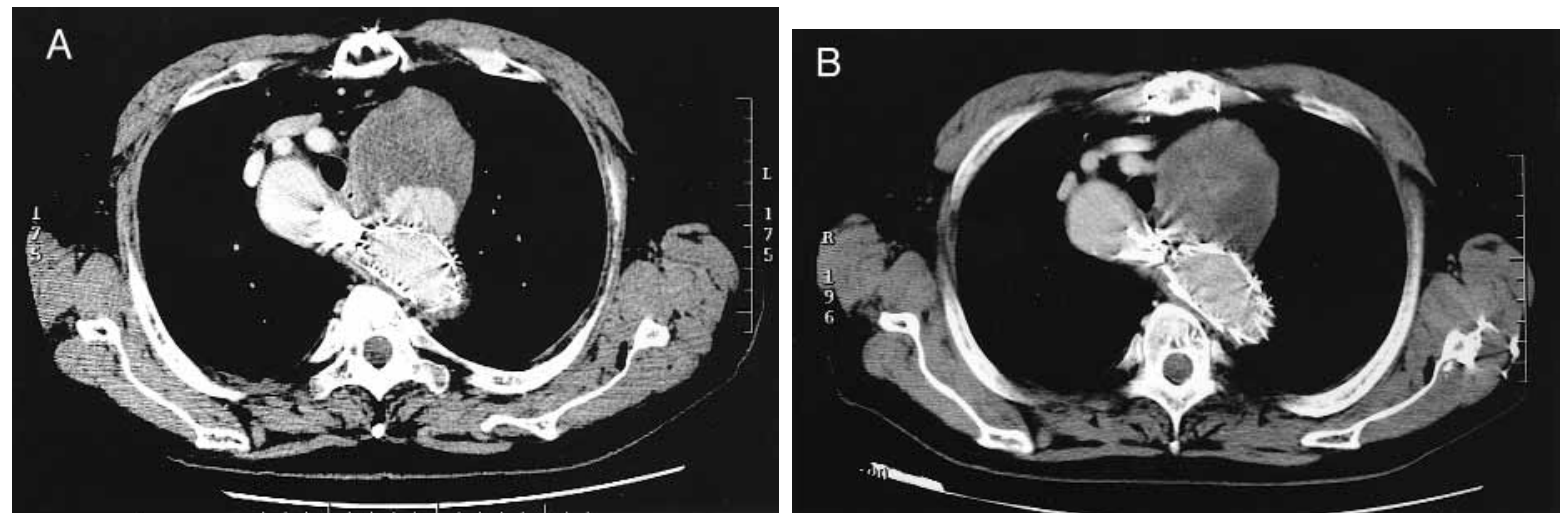

Figure 2. CT 2 weeks after the intervention (A) showed a small leakage from the stent-graft, although the leakage disappeared from CT 2 months after the intervention (B).

These conditions were suitable for endovascular stent-graft repair in this patient. This is the first patient treated with an endovascular stent-graft for thoracic aortic aneurysm with a right-sided aortic arch. Endovascular stent-graft repair is an alternative in the treatment of this entity.

\section{References}

1. Dake MD, Miller DC, Mitchell RS, Semba CP, Moore KA, Sakai T. The "first generation" of endovascular stent-grafts for patients with aneurysms of the descending thoracic aorta. J Thorac Cardiovasc Surg. 1998;116:689-704.
2. Hastrier AR, D’Cruz IA. Right-sided aorta. Part 1. Occurrence of right aortic arch in various types of congenital heart disease. Br Heart J. 1965;28:722-5.

3. Imagawa H, Kadoba K, Taniguchi K, Sawa Y, Takahashi T, Fukushima $\mathrm{N}$, et al. Saccular aneurysm in the right-sided aortic arch: a successfully corrected case. J Vasc Surg. 1997;25:941-4.

4. Caus T, Gaubert JY, Monties JR, Moulin G, Mouly A, Cornen A, et al. Right-sided aortic arch: surgical treatment of an aneurysm arising from a Kommerell's diverticulum and extending to the descending thoracic aorta with an aberrant left subclavian artery. Cardiovasc Surg. 1994;2:110-3. 